\title{
Characterization of acoustic emission signals from particulate filled thermoset and thermoplastic polymeric coatings in four point bend tests
}

\author{
Y. Xu* and B. G. Mellor \\ Materials Research Group, Engineering Sciences, University of Southampton \\ Highfield, Southampton, SO17 1BJ, UK
}

\begin{abstract}
Four point bend tests were conducted on two previously characterized particulate filled thermoplastic and thermoset polymeric coatings, the acoustic emission (AE) method being utilized to monitor the damage progress during the tests. Different damage mechanisms operating in the polymeric coatings can be recognized by the different amplitude range of the $\mathrm{AE}$ signals emitted. AE can be used to assess the relative strength of particle/matrix bonds in particulate filled polymeric coatings.
\end{abstract}

Keywords: Filled polymeric coating; Acoustic emission; Four point bend test; Damage mechanisms

\footnotetext{
* Corresponding author: Tel.: +44 1895 265883; fax: +44 1895269861.

Email address: yanmeng.xu@brunel.ac.uk (Y. Xu)

${ }^{+}$Current address: School of Engineering and Design, Brunel University, Uxbridge UB8 3PH, UK.
} 


\section{Introduction}

Acoustic emission (AE) signals are widely used to monitor a variety of failure modes for composite materials including matrix cracking, micro-crack initiation and growth, fracture of inclusions in a material, debonding, plastic deformation etc [1-9]. However, there are no studies correlating AE signals with the failure mechanisms in both particulate filled thermoset and thermoplastic coatings. In this study the AE technique was used to examine in-situ time controlled and stress dependant damage progress during four point bend tests on both particulate filled thermoset and thermoplastic polymeric coated samples. The different damage mechanisms in the thermoset and thermoplastic coatings were characterized by correlating the $\mathrm{AE}$ signals obtained during the test process with the loading conditions.

\section{Experimental}

\subsection{Characterization of polymeric coatings}

Figures 1 (a) and (b) show the cross sections of the thermoset coating T-34B and thermoplastic coating F4001B. T-34B is a $250 \mu \mathrm{m}$ thick epoxy coating deposited on a $100 \mu \mathrm{m}$ thick phenolic primer layer. The coating matrix is filled with $20 \%$ by volume of alumina particles, and the primer layer is filled with approximately $35 \%$ of calcium silicate particles. Cavities were observed on the metallographically prepared cross section of the coating as a result of detachment of filler particles from the matrix during the polishing process. This indicates that the bonding between the fillers and the coating matrix was relatively weak. The F-4001B coating consisted of a $1000 \mu \mathrm{m}$ thick fluoropolymer on a $200 \mu \mathrm{m}$ thick primer layer. The coating matrix contained $2 \%$ by volume of fluorite particles and the primer layer held $12 \%$ silicon carbide particles. The fillers in the primer are not evenly distributed and not well bonded, which causes microcracks and voids in the primer layer.

From the volume fraction of filler particles present in the coating and primer and the thicknesses of the coating matrix and the primer layers, it can be deduced that the number of the filler particles in both coating samples was about the same. 

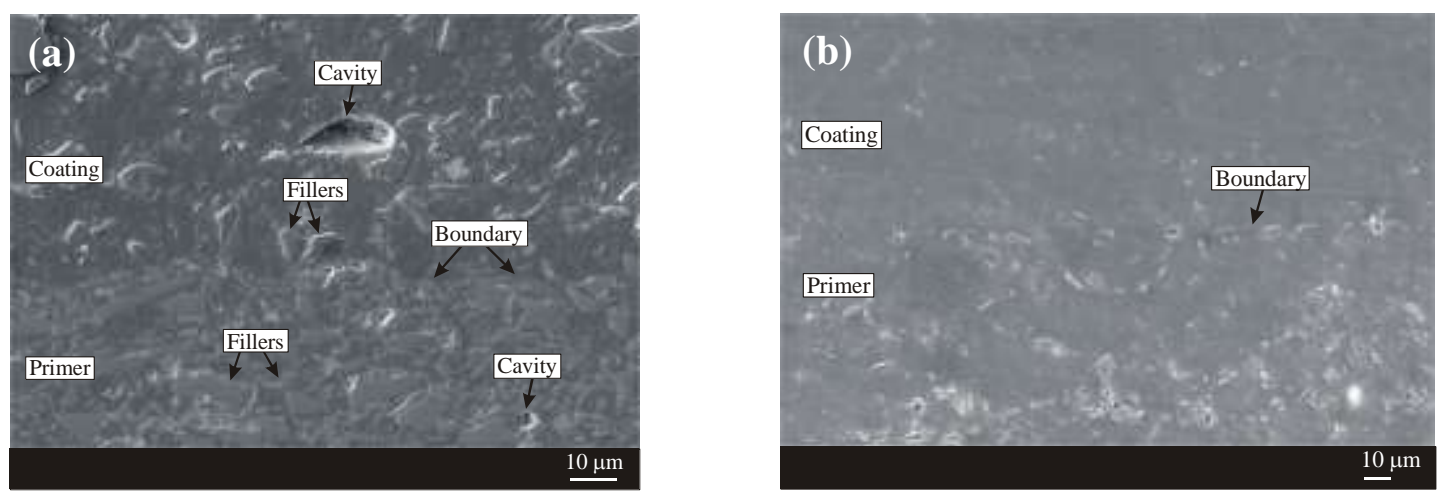

Figure 1 SEM images of cross sections of (a) the thermoset coating T-34B and (b) the thermoplastic coating F-4001B (b).

\subsection{Experimental procedure}

The four point bend tests were conducted on an Instron 8874 Servohydraulic testing machine and Vallen AE-Suite equipment was used to monitor the in-situ cracking of the specimen. A pair of ceramic rollers with a span of $40 \mathrm{~mm}$ was fitted in the grooves on an aluminum block to support the test specimen and another pair of rollers with a span of $20 \mathrm{~mm}$ was put on the steel substrate of the sample to support the load applied from the Instron machine. The coating was loaded so that it was subjected to tensile stress. The load was applied at a constant crosshead speed of $0.3 \mathrm{~mm} / \mathrm{min}$, the distance that the crosshead (load cell of the Instron machine) moved was fixed at $4 \mathrm{~mm}$ for all the specimens tested. The size of the coated samples was $18 \mathrm{~mm} \mathrm{x}$ $90 \mathrm{~mm}$ to fit the four point bend test rig and the AE sensors used. The steel substrates of all the coating samples were machined to an identical thickness of $1.5 \mathrm{~mm}$.

As a large number of trial tests showed the background noise caused by friction between the ceramic rollers and the coating surface was lower for the thermoplastic coating F-4001B than for the thermoset coating T-34B, the threshold parameters in the signal processor unit (SPU) of the AE system were set at 52 $\mathrm{dB}$ for the $\mathrm{T}-34 \mathrm{~B}$ coating and at $45 \mathrm{~dB}$ for the F-4001B coating in order to filter these unwanted AE signals. One PZT transducer was mounted on each end of the samples.

\section{Experimental results and discussion}

Figures 2 (a) and (b) present the coated specimens T-34B and F-4001B after testing. There were a few macrocracks and microcracks on the surface of coating T-34B, plus delaminations between the coating and the steel substrate. The chipping noted at the edge of the T-34B specimen (Figure 2 (a)) was caused by the cutting process when preparing these sample blanks, even though the cutting force applied to the top surface 
of the coating was small. This indicates that the coating was brittle, cracked easily and the bonding between the coating layer and the steel substrate was very weak, i.e. delamination is quite easy in this coating system. However, the deformed coating sample F-4001B appeared to have no cracks on the coating surface or delaminations between the coating and the steel substrate. This demonstrates that the thermoplastic coating F-4001B is more ductile and exhibits better bonding between the coating and the substrate than the thermoset coating T-34B under the same applied stresses.

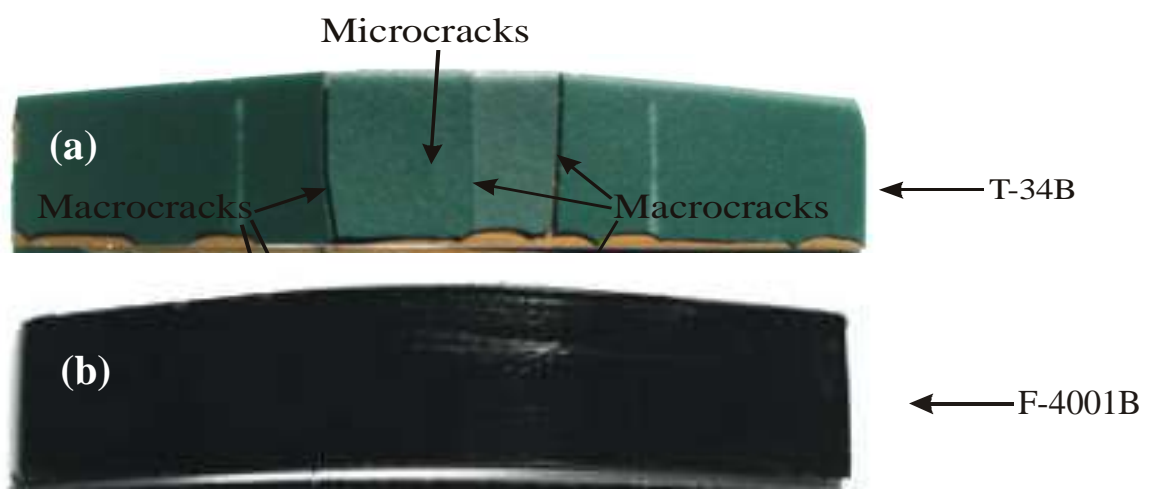

Figure 2 Surfaces of the coated specimens of the thermoset coating T-34B (a) and the thermoplastic coating F-4001B (b) after testing.

Figures 3 (a) to (f) show the AE results for coated samples T-34B and F-4001B during four point bend tests. The yield loads of both the coated samples determined from Figures 3 (a) and (b), were similar at approximately $0.8 \mathrm{kN}$. This indicates that the steel substrate dominated the overall strength of the samples tested due to its Young's modulus being much higher than that of the polymeric coating materials. The total number of AE events registered during the four point bend tests was larger by an order of magnitude for T34B. AE events commenced from the very start of loading for F-4001B while for T-34B AE events started after some elastic deformation of the steel substrate.

Figures 3 (c) and (d) show the amplitude distributions and the cumulative energies of the AE events recorded by the two sensors on each coating during the test. Since there were no cracks observed on the thermoplastic coating F-4001B through the whole test duration and the only source of all the AE signals collected was from debonding between the fillers and the coating matrix and primer, it can be deduced that the amplitude of the AE events for debonding fillers was below $60 \mathrm{~dB}$, as shown in Figure 3 (d). For T-34B three major groups of AE signals can be separated according to the amplitude levels: i) amplitude below 60 $\mathrm{dB}$, ii) amplitude between 60 and 75, and iii) amplitude above $75 \mathrm{~dB}$. AE signals above $60 \mathrm{~dB}$ were 
considered to be mainly due to initiation and growth of cracks in the coating and delamination between the coating and the steel substrate. As the cracks in the coating must have occurred prior to the delaminations and the delaminations take place towards the end of the test process it can thus be concluded that the high amplitude signals above $75 \mathrm{~dB}$ were caused by the initiation and growth of the cracks, whereas the AE signals between 60 to $75 \mathrm{~dB}$ were caused by delamination. Similar results have been reported by Miguel et al. [1]. It should be noted that two high amplitude AE events occurred for T-34B before yield of the steel substrate took place.

Low values of cumulative energy, i.e. $200 \mathrm{eu}$ and $700 \mathrm{eu}$, were recorded by the two sensors at the end of the test for the thermoplastic coating F-4001B, however, much higher values of 12E4 eu and 13E4 eu were recorded for the thermoset coating T-34B. The cumulative energy values from T-34B were thus about 300 times higher than for F-4001B. The total number of the AE events emitted from the coating T-34B was about 10 times greater than that from the coating F-4001B, although the total number of particles in both of the two coatings was about the same. It is believed that the greater number of the AE events in T-34B was mainly caused by the delaminations, and the higher cumulative energies were incurred from the high amplitude AE events which were associated with the delaminations, and the initiation and propagation of the cracks. The position of the AE events of amplitude greater than $75 \mathrm{~dB}$ shown in Figure 3 (e) was found to correspond to the location of cracks in the thermoset coating T-34B. This result again indicates that the high amplitude AE signals are associated with the cracking mechanism. Figure 3 (f) presents the locations of the AE signals collected for the thermoplastic coating F-4001B, which were randomly distributed along the span of the coating.

As noted earlier two high amplitude AE events (amplitude > 75dB) occurred during the elastic deformation of the steel substrate for T34B. These high amplitude AE events arise from the formation of the macrocracks seen on this coating. This occurs at relatively low strains as the coating was brittle and bonding between the coating and the substrate was weak. Although the stiffness of the sample will be reduced by the above damage mechanisms as they started to develop when the steel was behaving elastically with a Young's modulus of $207 \mathrm{GPa}$, which is nearly one order of magnitude greater than that of the coating, no sudden load drop during the test was observed associated with the formation of these cracks. Load drops might be expected to occur if the coating first cracked during the plastic derformation of the steel substrate [9]. 


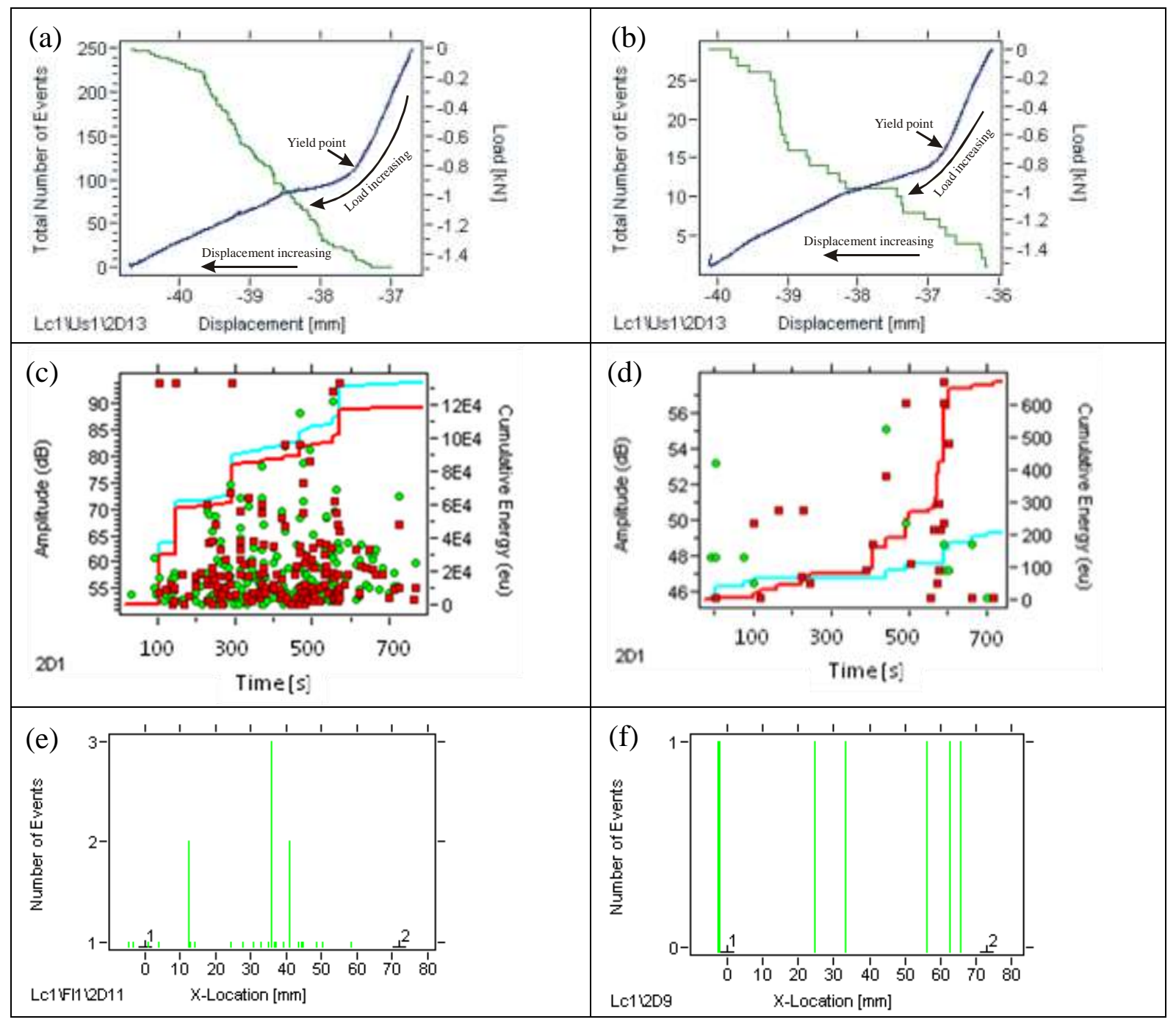

Figure 3 Experimental results of AE signals during four point bend tests for the coatings T-34B and F-4001B. (a) T-34B; (b) F-4001B; (c) T-34B; (d) F-4001B; (e) T-34B, filtering condition: Amplitude < 75 dB removed; (f) F-4001B.

\section{Conclusions}

In this study, the different damage mechanisms that occur during four point bend tests on thermoset and thermoplastic coatings were investigated. The initiation and propagation of cracks emit higher amplitude AE signals than the delamination of the coating layer from the substrate, whilst the debonding mechanism between the fillers and the surrounding materials emits the lowest amplitude of AE signals. AE signals can be used to assess the relative bond strength between fillers and matrix as well as to locate the position of cracks by applying appropriate filters to the AE system. 


\section{REFERENCES:}

[1] Miguel JM, Guilemany JM, Mellor BG, Xu YM, Acoustic Emission Study on WC-Co Thermal Sprayed Coatings, Mater Sci Eng. 2003; A352: 55 - 63.

[2] Park JM, Kong JW, Interfacial evaluation of electrodeposited single carbon fiber/epoxy composites by fiber fracture source location using fragmentation test and acoustic emission, Composites Science and Technology 2004; 64: 983-999.

[3] Sagar RV, Prasad BKR, AE energy release during the fracture of HSC beams, Magazine of Concrete Research 2009; 61: 419-435.

[4] Jerabek M, Major Z, Filler/matrix-debonding and micro-mechanisms of deformation in particulate filled polypropylene composites under tension, Polymer 2010; 51: 2040-2048.

[5] Oskouei AR, Ahmadi M, Acoustic Emission Characteristics of Mode I Delamination in Glass/Polyester Composites, J of Composite Mater 2010; 44: 793-807.

[6] Yang L, Zhou Y, Wavelet analysis of acoustic emission signals from thermal barrier coatings, Trans. Nonferrous Met. SOC. China 2006; 16: 270 - 275.

[7] Bentahar M, Guerjouma R, Monitoring progressive damage in polymer-based composite using nonlinear dynamics and acoustic emission, $\mathrm{J}$ of the Acoustical Society of America 2009; 125: $39-44$.

[8] Károly Renner, János Móczó, Quantitative determination of interfacial adhesion in composites with strong bonding, European Polym. Journal 2010; 46: 2000 - 2004.

[9] Xu Y, Mellor BG. Application of acoustic emission to detect damage mechanisms of particulate filled thermoset polymeric coatings in four point bend tests. Surface \& Coatings Technology 2011;

205:5478-82. 